\title{
INNOVACIÓN: CLAVE PARA EL ÉXITO DE LA GESTIÓN EMPRESARIAL EN LA MICRO, PEQUEÑA $Y$ MEDIANA EMPRESA
}

\author{
ALBA MARITZA GUERRERO SPÍNOLA DE LÓPEZ \\ Universidad de San Carlos de Guatemala \\ aguerrero@ing.usac.edu.gt
}

\begin{abstract}
RESUMEN
En estos tiempos de constantes y vertiginosos cambios, en la llamada era de las Sociedades del Conocimiento es indispensable que las Micro, Pequeñas y Medianas Empresas (MIPYMES) comprendan que es necesario innovar para enfrentarse al desafí y oportunidad que enfrentan los países en vías de desarrollo para lograr salir de la pobreza. El artículo parte de la definición del concepto de innovación y los tipos de innovación que existen, remarcando que la innovación es más que tecnología. Posteriormente se mencionan los factores que determinan el éxito de la innovación, así mismo, se presentan los modelos de innovación de acuerdo con diversos autores. Adicionalmente, para lograr una gestión de innovación exitosa es indispensable el establecimiento de un Sistema Nacional de Innovación el cual facilitará que las MIPYMES coordinen con cada uno de los actores: Estado a través de las Instituciones de Gobierno, Centros de Investigación y las Instituciones de Educación Superior con el objeto de que cada actor contribuya a lograr procesos de innovación exitosos. Finalmente se considera la dependencia entre los términos de innovación y estrategia, por lo cual se describen los tipos de estrategia empresarial resaltando la importancia de la estrategia basada en la innovación como factor clave para el éxito empresarial.
\end{abstract}

PALABRAS CLAVE: INNOVACIÓN, ESTRATEGIA, ESTRATEGIA DE INNOVACIÓN, SISTEMA NACIONAL DE INNOVACIÓN.

\begin{abstract}
In the so-called Knowledge society, it is essential for Micro, Small and Medium Enterprises (SMEs) to innovate in order to grow - a challenge and an opportunity faced by developing countries to overcome poverty. This article is, first, based on the definition and typology of innovation, to point out that innovation is more than technology, and, then, mentions the factors that determine the success of innovation, by showing different models of innovation according to various authors. To achieve a successful innovation management model, it is essential to establish a National Innovation System which will facilitate MSMEs coordination with all other actors: the State through Government Institutions, Research Centers and Higher Educational Institutions in order to help each participant achieve successful innovation processes. Finally, the relation between innovation and strategy is considered by analyzing the types of business strategy and by highlighting the importance of strategy based on innovation as the key to business success.
\end{abstract}

KEYWORDS: INNOVATION, STRATEGY, INNOVATION STRATEGY,THE NATIONAL INNOVATION SYSTEM.

\section{INTRODUCCIÓN}

La cada vez más rápida obsolescencia de los procesos y los productos que caracteriza al escenario competitivo mundial, ha extendido y popularizado la idea de que la innovación es la clave para el éxito de las empresas. Entonces, ¿por qué razón las empresas no encaminan sus esfuerzos para lograr productos y servicios diferenciados e inno- 
vadores que logren atraer a los consumidores y les permitan mantenerse en el mercado global?, algunos mitos que impiden a las empresas innovar de acuerdo con su tamaño son:

a) La innovación es sinónimo de inversión y las MIPYMES no cuentan con dichos recursos, es decir la innovación es un proceso que requiere mucha inversión en investigación y desarrollo de productos que únicamente las grandes empresas y sobre todo las transnacionales tienen la capacidad de realizar.

b) Las MIPYMES no cuentan con recursos humanos especializados para generar ideas creativas.

c) Es difícil incursionar en mercados internacionales y sobre todo mantenerse con productos novedosos, pues no se cuenta con la capacidad instalada para satisfacer una posible demanda.

Si innovar se presenta como la solución para que las MIPYMES subsistan en un mundo competitivo, entonces ¿qué deben conocer los directivos sobre este tema y cómo hacer que sus empresas aprovechen las ideas creativas del talento humano que las conforman?

\section{El concepto de innovación}

Existe diferente literatura que aborda el tema de la innovación considerando que esta se encuentra relacionada con la tecnología y los aspectos más complicados de una empresa como son la organización, estructura, procesos, entre otros (Domenech, 2009; West \& Farr ,1990; Schumpeter, 1912; Tanaka, Glaude \& Gault 2005). Cada autor toma su postura respecto de la concepción que le dará al tema, pero la mayoría de autores coinciden en que la innovación es un proceso empresarial capaz de convertir una buena idea en un producto, servicio, estrategia, modelo, que sea aceptado y valorado exitosamente por un segmento de mercado determinado.
La innovación es un término que ha tenido incidencia en el campo económico, empresarial e industrial, su concepción ha variado conforme su campo de aplicación, revisemos algunos conceptos.

Domenech (2009), en una entrevista para la revista Innova, menciona que para él innovar es crear productos que hagan la vida más fácil, exige la conciencia y el equilibrio para transportar las ideas, del campo imaginario o ficticio al campo de las realizaciones e implementaciones.

Se considera además que la innovación es la secuencia de actividades por las cuales un nuevo elemento es introducido en una unidad social con la intención de beneficiar la unidad, una parte de ella o a la sociedad en su conjunto. El elemento no necesita ser enteramente nuevo o desconocido a los miembros de la unidad, pero debe implicar algún cambio discernible (West \& Farr ,1990).

Por su parte, Schumpeter (1912), quien introdujo por primera vez el concepto de innovación, mencionó que esta se refiere a una aplicación específica de la tecnología desarrollada por el proyecto y que tiene la característica de haber sido colocada exitosamente en el mercado. La innovación crea valor para quienes la utilicen; existe un estado de estancamiento empresarial denominado circuito económico, y un estado de crecimiento, al que llama evolución. El paso del circuito a la evolución se efectúa por medio de las innovaciones, las cuales se constituyen en el motor del crecimiento.

Tanaka, Glaude y Gault (2005) definen la innovación como:

La introducción de un nuevo significativamente mejorado, producto (bien o servicio) de un proceso, de un nuevo método de comercialización o de un nuevo método organizativo, en las prácticas intermedias de la empresa, la organización del lugar de trabajo o las relaciones exteriores (p. 16).

Schumpeter (1939) introduce, por una parte, las diferencias entre invención, definida como la gene- 
ración de una nueva pieza o conocimiento; innovación, como la traducción de la Innovación más Desarrollo (I+D) anteriores, en un nuevo producto/ proceso que llega al mercado; y difusión como la imitación de la innovación al ser adoptado por un número grande de competidores.

Los autores citados coinciden en que la innovación es un cambio o mejora que puede tener un producto y/o servicio luego de realizarse un proceso de investigación y desarrollo. Innovar no es sinónimo de crear algo nuevo, sino de mejorar o adicionar algún factor a un producto y/o servicio que ya existe en el mercado.

La innovación es, por lo tanto, un proceso de gestión empresarial que consiste en identificar oportunidades del mercado que conlleven a la introducción de nuevos productos, nuevos servicios, nuevos procesos o a la modificación significativa de los actuales productos y procesos (Martín-Hidalgo, 2009). La innovación es un reto estratégico para las empresas que requiere la generación constante de buenas ideas que deberán convertirse en productos y servicios con una alta probabilidad de éxito comercial.

Innovar es técnica, método, proceso y control. Pero sobre todo es ganas, empuje, coraje, trabajo en equipo, pasión, arte, diseño, meditación, intuición (Ponti, 2010). Evidentemente existen innovaciones que brotan en un instante de genialidad, sin embargo, la mayoría de innovaciones requiere de una búsqueda constante de oportunidades, de un trabajo tesonero, de una mezcla entre el orden, la disciplina, la capacidad artística y la creatividad.

Recientemente, Drucker (2002) lanzó la idea de que las empresas compiten ya no con productos sino con modelos empresariales, los cuales nacen de la innovación y son más competitivos cuanto más intensa sea esta. La innovación recae en las partes de la cadena que aportan más valor al sector y a las empresas.

Por lo tanto, innovar se asocia con un proceso empresarial que convierte un conjunto de buenas ideas en productos, servicios, procesos, estrategias, métodos que son valorados exitosamente por el mercado y que contribuyen a mejorar las condiciones de un sector de la economía y que beneficia en forma positiva a la sociedad en general. Además de producir rentabilidad a la empresa, aporta bienestar.

Así mismo, el concepto de innovación ha sido tradicionalmente relacionado con el desarrollo de nuevos productos, procesos de producción y tecnologías, ligadas directamente con las grandes empresas que dentro de su estructura organizativa contemplan el Departamento de Investigación y Desarrollo, empresas como Google, Apple, Facebook, Nokia, General Electric, Microsoft que invierten cantidades millonarias en la generación de nuevos conceptos.

Sin embargo, como menciona Ponti (2010), absolutamente todas las empresas pueden innovar y cada vez están más obligadas a hacerlo, debería ser el pan de cada día. La innovación debe verse como un proceso dinámico, un proceso de gestión empresarial basado en la gestión de personas, de información, de conocimiento y recursos tanto financieros como tecnológicos.

Pero innovar ¿es sinónimo de creatividad?, es importante aclarar la diferencia que existe entre los términos de innovación, invención y creatividad. La creatividad es la capacidad humana que todos los seres humanos poseemos en mayor o menor medida asociada al proceso de generación de ideas originales. La invención está relacionada con el desarrollo práctico de prototipos u objetos novedosos.

La innovación, por otro lado, como se ha mencionado anteriormente, es un proceso empresarial, capaz de convertir una buena idea (o un conjunto de buenas ideas) en un producto, servicio, estrategia, método, proceso, entre otros, que sea valorado exitosamente por un público determinado (Ponti, 2010). La innovación va más allá, supone la colocación en el mercado de productos y/o servicios valorados por los clientes. A pesar 
de presentarlos como conceptos diferenciados, tanto la creatividad como la invención forman parte del proceso integral de innovación.

La innovación suele venir asociada a la idea de "romper con las reglas del juego", dicho de otra forma, supone la ruptura de paradigmas o el cuestionamiento profundo de sistemas que hasta el momento han sido plenamente aceptados.

No es imposible lanzarse a la búsqueda del emprendimiento innovador, basta que se tenga claridad ¿por qué se quiere innovar?, ¿cómo se hará?, ¿quién lo hará?, que se tenga la capacidad de romper esquemas, moldes, paradigmas y sobre todo contar con personas que hagan las cosas de forma diferente. Se verán ahora los tipos de innovación a los cuales una empresa puede apostar.

\section{Tipos de Innovación}

El término innovación se usa de manera diferente de acuerdo con el nivel de análisis empleado. Las distinciones más útiles son las que definen los tipos de innovación como técnica, administrativa y organizacional-ambiental.

Damampour (1987) y Damampour \& Evans (1998) se refieren a las innovaciones técnicas como aquellas que ocurren en los sistemas técnicos de una organización y que están directamente relacionados con la actividad primaria de trabajo de dicha organización. Una innovación técnica puede ser la implementación de una idea para un nuevo producto o un nuevo servicio, o la introducción de elementos nuevos en las operaciones de producción o servicios de una organización.

Schumpeter (1939) distingue cinco formas de innovación: de producto, de proceso, de nuevas materias primas, de nuevos mercados y de reorganización industrial. La innovación tecnológica en productos y procesos (ITPP) se refiere a la implementación tecnológica de nuevos productos y procesos o a mejoras significativas en estos, ya sea como resultado de la difusión de cono- cimientos tecnológicos o de inversiones en I+D que generan novedades a nivel de la empresa.

La innovación en productos puede tomar dos formas: a) un producto tecnológicamente nuevo, es decir, un producto cuyas características tecnológicas difieren significativamente de las correspondientes a los productos anteriores. Este puede implicar tecnologías radicalmente nuevas o la combinación de tecnologías existentes con nuevos usos, así como derivarse del uso de un conocimiento nuevo; b) un producto existente tecnológicamente mejorado. Esto se puede dar por el uso de componentes o materiales de mejor desempeño, o por un producto complejo compuesto por un conjunto de subsistemas técnicos integrados que pudo haber sido mejorado a través de cambios parciales. Las innovaciones en producto implican la presentación de productos/servicios nuevos o mejorados para los clientes (Martín-Hidalgo, 2009).

La innovación de producto no tiene porqué estar únicamente basada en el desarrollo de competencias tecnológicas genéricas. En el producto, existen una serie de elementos que pueden ser objeto de mejora profunda como por ejemplo: complementos, estilo, características físicas, calidad, medidas. En el cliente, se podría trabajar sobre el uso del producto, la percepción del producto, o incluso de la empresa, el segmento elegido, el nivel de precios, entre otros.

La innovación en procesos es la adopción de métodos tecnológicos nuevos o mejorados, incluyendo los métodos de distribución, y puede comprender cambios en equipos, en la organización de la producción, o consistir en una combinación de los anteriores. Puede, también, generarse de la aplicación de un nuevo conocimiento. Estos métodos pueden ser aplicados para producir o despachar productos tecnológicamente mejorados.

Tradicionalmente los estudios sobre innovación de procesos se centran en el proceso productivo. A partir de los años 80, con la aparición de los programas de calidad total, se inicia la aplicación 
de técnicas de mejoras de procesos a todos los niveles de la empresa: productivos, comerciales, administrativos, de soporte, entre otros. Impulsos como el de Motorola o General Electric que han utilizado los enfoques "6 sigma", los cuales se basan en herramientas estadísticas y que adicionalmente han tenido una clara visión de negocio, han demostrado que la innovación en procesos va más allá del proceso productivo.

Según Pujol (2005), la innovación de procesos es el proyecto de cambio profundo de un proceso multifuncional con el objetivo de mejorar de forma significativa tanto los resultados intermedios como los outputs finales en términos de variables como la calidad el costo, la flexibilidad o el plazo de realización.

Para Martin-Hidalgo (2009), las innovaciones en procesos son novedades o mejoras de los procesos de producción y provisión del servicio. Estas se pueden dividir en innovaciones en los procesos de producción e innovaciones en los procesos de distribución y entrega (provisión), es la implementación de un método de producción o distribución nuevo o con un alto grado de mejora. Esta incluye mejoras importantes de técnicas, equipo y/o software.

En cuanto a las innovaciones administrativas Damampour (1987) y Damampour \& Evans (1998), las definen como aquellas que ocurren en el sistema social de una organización, la implementación de una nueva manera de reclutar personal, distribuir recursos o estructurar tareas, autoridad y recompensas. Comprenden innovaciones en la estructura organizacional y en la dirección de las personas. Una innovación organizacional es la implementación de un nuevo método de organización aplicado a las prácticas del negocio, al lugar de trabajo o a las relaciones externas de la empresa.

Las innovaciones administrativas son consideradas menos efectivas porque son menos observables, más complejas de implementar y relativamente menos ventajosas (Damampour \& Evans, 1984; Evans \& Charles, 1998). De ahí que las innovaciones tecnológicas sean percibidas como más efectivas que las administrativas o las de vínculo con el ambiente. También podría desprenderse de lo anterior que los empresarios tienden a percibir en mayor grado las tecnológicas y por ello a adoptarlas más.

Una innovación de marketing es la implementación de un nuevo método de comercialización que entraña importantes mejoras en el diseño del producto o en su presentación, o en su política de emplazamiento (posicionamiento), promoción o precio (Martín-Hidalgo, 2009).

Otra forma de clasificar la innovación para Martín-Hidalgo (2009) se da por el nivel de impacto en la competitividad de la empresa en: a) radical o estratégica, que es aquella que contribuye a que la empresa compita a un mediano y largo plazo, generalmente asociada con el lanzamiento al mercado de nuevos productos y nuevos servicios. Demanda cambios tecnológicos radicales en la empresa; b) incremental, que es aquella que se realiza en los productos, servicios o procesos existentes en la empresa con el fin de mejorar su desempeño en el mercado. Por lo general contribuye a que la empresa pueda competir en el corto y mediano plazo.

Las innovaciones incrementales tienen que ver con la mejora y optimización de los productos, servicios, procesos de producción, modelos organizacionales, estrategias, entre otros, que conducen a la obtención de productos y servicios más avanzados, pero con el mismo concepto de aquellos que los preceden. La innovación radical proviene, más que de optimizar lo que ya se tiene, de imaginar aquello que no ha sido nunca. Consiste en repensar las realidades de forma diferente a la que se acostumbra en la experiencia previa, desde otra perspectiva, buscar opciones novedosas sin prejuicios, paradójicamente dichas transformaciones son producidas por personas externas al sector, es decir, personas que no están acostumbradas a hacer las cosas de la forma tradicional (Llobet, 2006). 


\section{Factores que determinan la innovación}

El proceso de innovación está determinado por factores, entendiendo un factor como aquel elemento que puede condicionar una situación, volviéndose el causante de la evolución o transformación de los hechos. Existen factores que pueden determinar la capacidad innovadora a nivel mundial, por región, por país, por sector de la economía y por empresa.

Los factores que determinan la capacidad de innovar y emprender de un país se pueden agrupar en seis grandes grupos: los gobiernos, las empresas, el sistema educativo, las personas, los círculos sociales y la responsabilidad social. Los gobiernos son los encargados de la definición de las políticas y las leyes que faciliten a las empresas el proceso de innovación. Por su parte, las empresas son el escenario de la innovación. En el sistema educativo es donde se fijan las bases de la innovación a través de los centros de investigación. Las personas son los generadores de innovación, de ellas surgen las ideas, las motivaciones y las ambiciones que dan lugar a la innovación. Los círculos sociales son los vínculos que unen la diversidad de perspectivas de las personas y que potencian la innovación. Finalmente, la responsabilidad social, mientras tanto, posibilita una tipología de innovación que el mercado olvida: la innovación social.

Estos seis grandes grupos permiten elaborar un marco general que da consistencia a una agenda de innovación. Los gobiernos deben diseñar políticas pensando en el conjunto de su región, pero siempre deberán respetar un elemento fundamental: la innovación sostenible de una región no depende de sus políticas e instituciones, depende de sus empresas y personas. Por ello se debe fomentar una cultura innovadora.

A nivel de empresa, el éxito o fracaso de los procesos innovativos está determinado por factores que, de acuerdo con la concepción de varios autores como Díaz (1996), Hidalgo y Vizán (2008), se pueden clasificar como endógenos o exóge- nos. A continuación se presenta una breve descripción de los estos.

Según Díaz (1996) la estructura del mercado y las características del sector donde la empresa desarrolla su actividad son los factores determinantes de la innovación. Mientras que Hidalgo y Vizán (2008) indican que los factores que caracterizan el proceso de innovación se pueden dividir en internos y externos.

Los factores internos son resultado de actuaciones de la propia empresa y dependen de la capacidad de dirección para consolidar las dimensiones de la calidad y productividad (corto plazo), así como la posibilidad de crear a menor costo y más rápidamente que los competidores, tecnologías, competencias y aptitudes esenciales que generen productos o servicios innovadores (largo plazo).

Los factores externos están asociados al sector de actividad al que pertenece la empresa, al contexto institucional que la rodea y a las características de la política económica que le afecta.

Harris, Coles y Dickson (2000) mencionan como factores importantes la situación organizacional y el potencial tecnológico, el cual comprende las capacidades tecnológicas internas que la empresa tiene para vincularse con las oportunidades del mercado. En la teoría general a nivel de empresa individual, los factores que afectan el desarrollo del nuevo producto son: la competencia tecnológica, el tamaño de la empresa, el sector y la localización. El intercambio de información informal entre las empresas, es un factor que favorece el desarrollo de un nuevo producto así como las alianzas estratégicas como una forma de colaboración tecnológica con otras empresas son un factor que fortalecen a las empresas en el desarrollo de un nuevo producto.

Jaramillo, Lugones y Salazar (2001) indican que los factores que determinan los procesos innovativos se clasifican en: empresariales o microeconómicos, meso-económicos o de merca- 
do, y macro y meta económicos los cuales a su vez se sub dividen así:

- Empresariales o microeconómicos: capacidades de innovación, disponibilidad de personal capacitado, resistencia al cambio, deserción laboral, riesgo de innovar, período de retorno, costos de la innovación.

- Meso-económicos o de mercado: tamaño del mercado, estructura del mercado, comercialización, dinamismo del sector, respuesta de los consumidores ante productos y procesos nuevos, oportunidad para cooperar, oportunidad tecnológica, dinamismo tecnológico, necesidad de innovar, costos del financiamiento, disponibilidad de financiamiento facilidad para imitar las innovaciones.

- Macro y meta-económicos: información sobre mercados; información sobre tecnologías; legislación, normas, regulaciones, estándares, impuestos; instituciones públicas; instituciones relacionadas con ciencia y tecnología; infraestructura física; sistema de propiedad intelectual; legislación y regulaciones laborales; calidad de la formación básica de los trabajadores; costos de capacitación; calidad y disponibilidad de centros de formación.

Márquez y Pérez (2007) señalan que los factores que determinan la innovación se clasifican de acuerdo con siete enfoques diferentes (Tabla 1).

TABLA 1

\section{Factores determinantes de la innovación}

\begin{tabular}{|c|c|c|}
\hline Enfoque & Principales autores & Factores considerados \\
\hline $\begin{array}{l}\text { Economía industrial } \\
\text { (Estructura-Conducta-Resultados) }\end{array}$ & $\begin{array}{l}\text { Schmookler } \\
\text { Scherer } \\
\text { Schmalensee }\end{array}$ & $\begin{array}{l}\text { - Tamaño de mercado } \\
\text { - Estructura de mercado } \\
\text { - Régimen de apropiabilidad de resultados(imitación) } \\
\text { - Oportunidad tecnológica del sector } \\
\text { - Nivel de gasto Investigación y Desarrollo (I+D) }\end{array}$ \\
\hline Estructural & $\begin{array}{l}\text { Comisión Económica } \\
\text { para América Latina } \\
\text { Banco Interamericano } \\
\text { de Desarrollo }\end{array}$ & $\begin{array}{l}\text { - Marco regulatorio } \\
\text { - Marco institucional y social } \\
\text { - Condiciones políticas } \\
\text { - Condiciones macroeconómicas } \\
\text { - Acceso y funcionamiento de los mercados de factores } \\
\text { - Acceso y funcionamiento de los mercados de bienes y servicios }\end{array}$ \\
\hline Shumpeteriano & Schumpeter & $\begin{array}{l}\text { - Tamaño empresarial } \\
\text { • Concentración del sector } \\
\text { • Empresario innovador }\end{array}$ \\
\hline Recursos y Capacidades & $\begin{array}{l}\text { Hamel y Prahalad Teece } \\
\text { Galende y de la Fuente }\end{array}$ & $\begin{array}{l}\text { - Recursos tangibles } \\
\text { - Recursos intangibles } \\
\text { - Capacidades }\end{array}$ \\
\hline Evolucionista & $\begin{array}{l}\text { Nelson y Winter } \\
\text { Dosi }\end{array}$ & $\begin{array}{l}\text { - Historia de la empresa } \\
\text { - Oportunidades de innovación } \\
\text { - Incentivos para innovar } \\
\text { - Modalidad de aprendizaje que adoptan los agentes } \\
\text { - Rutinas de la organización }\end{array}$ \\
\hline Costos de transacción & Coase Williamson & $\begin{array}{l}\text { - Nivel de retorno esperado } \\
\text { - Tiempo de recuperación de la inversión }\end{array}$ \\
\hline $\begin{array}{l}\text { Interacción } \\
\text { Empresa-Universidad }\end{array}$ & $\begin{array}{l}\text { Universidad Politécnica } \\
\text { de Valencia-España } \\
\text { (CERES) }\end{array}$ & $\begin{array}{l}\text { - Interface para provocar interacciones entre diferentes agen- } \\
\text { tes de innovación ubicados en el entorno tecnológico, acadé- } \\
\text { mico, empresarial y financieros }\end{array}$ \\
\hline
\end{tabular}

Fuente: Marquez y Pérez, 2007. 


\section{MODELOS DE INNOVACIÓN}

Partiendo de que un modelo representa la realidad por medio de abstracciones o explicaciones de esta, se presenta a continuación los distintos modelos que diversos autores han planteado para explicar o representar el concepto de innovación:

El primer modelo a considerar es el presentado por Afuah (2009) quien distingue entre los modelos estáticos y los modelos dinámicos de innovación. Los modelos estáticos explican los diferentes factores que afectan la capacidad innovadora de las organizaciones en un determinado momento histórico. Por su parte, los modelos dinámicos, toman una visión longitudinal de la innovación y exploran la evolución de los procesos innovadores de las empresas.

El segundo modelo a considerar es el introducido por Albernathy \& Clark (1985). Este modelo sugiere que existen dos tipos de conocimiento que señalan una innovación: tecnológico y de mercado. Por tanto, las capacidades tecnológicas de la empresa pueden estar obsoletas, pero no sus capacidades de mercado. De este modo la empresa puede utilizar sus capacidades de mercado para mantener su ventaja sobre las empresas entrantes. Así, el modelo clasifica las innovaciones de acuerdo con su impacto en el conocimiento existente sobre el mercado y sobre la tecnología.

Ambos autores definieron la innovación como la introducción inicial de mercado de un nuevo producto o proceso, cuyo diseño se aparta radicalmente de la práctica anterior. Por lo tanto, la importancia competitiva de una innovación dependerá de: lo que le da el valor agregado y su aplicabilidad a las competencias de la empresa. Además consideran la posición competitiva de una empresa en términos de variedad de dimensiones. Se asume que los productos no son homogéneos y que las empresas compiten por ofrecer productos que pueden ser diferentes en muchos aspectos como: rendimiento, fiabilidad, disponibilidad, facilidad de uso, apariencia estética e imagen, entre otros, sin menospreciar el costo inicial.

Una empresa obtiene ventaja competitiva cuando alcanza una posición en una de estas dimensiones o cuando logra una combinación en varias, que son valoradas por los clientes y superan a los competidores.

FIGURA 1

Mapa transiliencia Modelo de Albernathy-Clark

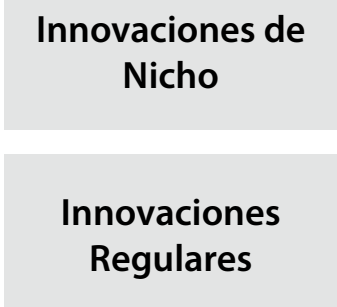

Innovaciones Arquitectónicas

Fuente: Izque (2005).

En este modelo se divide las innovaciones en dos dimensiones representadas a través de una matriz con cuatro celdas llamada "Mapa de transiliencia", que es una combinación de las palabras transitoria y resistencia, la cual ilustra cómo las innovaciones de productos diferentes afectan la situación competitiva en una industria determinada. El Mapa de transiliencia muestra la capacidad de una innovación y la influencia de los recursos existentes en la empresa, así como las habilidades y conocimientos. Se centra en dos perspectivas distintas, la primera se enfoca en cómo las nuevas tecnologías y las actividades de fabricación están organizadas, mientras que la segunda perspectiva relaciona las actividades necesarias que la empresa debe implementar en función de los mercados y clientes. La relación entre cada dimensión sugiere cuatro tipos de innovación:

a) Innovación revolucionaria: Se invierte en nueva tecnología y en innovación, se re- 
quiere una agresiva estrategia de mercadeo para cambiar reglas del juego y posicionarse competitivamente. Se aplica a mercados y clientes, no siempre tienen un impacto en la competencia.

b) Innovación regular: implica un cambio basado en la reconocida competencia técnica y de producción, se aplica a mercados y clientes, el objetivo es alcanzar altos volúmenes de producción y el uso de economías de escala para reducir costos y mejorar los productos. Se busca mejorar la calidad, las características de los productos y eliminar cuellos de botella en el proceso productivo.

c) Creación de un nicho: La apertura de nuevas oportunidades de mercado a través del uso de la tecnología existente es fundamental para este tipo de innovación, el efecto sobre los sistemas de producción y técnico es de conservar y fortalecer los diseños establecidos. Puede aparecer como la introducción de nuevos productos, detalles técnicos e incluso los cambios tecnológicos. Los cambios de basan en las competencias técnicas establecidas y la capacidad de mejorar su aplicabilidad en los segmentos de mercados emergentes. La fabricación debe ser rápida, sensible, ofrecer un servicio de respuesta ágil entregando el producto oportunamente.

d) Innovación arquitectónica: la esencia de esta innovación la constituye el uso de nuevos conceptos en tecnología para establecer nuevos vínculos de mercado. Define la configuración básica de productos y procesos estableciendo programas técnicos y de mercadeo que orientan el desarrollo de la empresa.

El tercer Modelo es el presentado por Henderson y Clark (1990): sugirieron que las innovaciones están hechas de componentes que están interconectados y que desarrollarlas requiere de dos tipos de conocimientos: conocimientos de los componentes y conocimiento de los vínculos; dentro de el de componentes llamaron a otro conocimiento arquitectural (ver figura 2).

Una innovación puede impactar un componente de conocimiento o arquitectural o ambos, con diferentes consecuencias para la empresa. De allí se derivan cuatro tipos de innovación: Si la innovación mejora los conocimientos del componente y el conocimiento arquitectural, es incremental; si destruye el conocimiento arquitectural y el del componente, es radical; si destruye el conocimiento arquitectural y se mejora el conocimiento del componente, la innovación es arquitectural; y si se destruye el conocimiento del componente y se mejora el conocimiento arquitectural la innovación es modular.

El cuarto modelo a considerar lo constituye el Modelo de innovación lineal (teoría neoclásica). El proceso de transferencia de los principios científicos a conocimientos útiles para la producción industrial es en esencia secuencial, comprende fases lineales que deben sucederse. La aparición de nuevas tecnologías sigue una secuencia temporal bien definida que comienza con actividades de investigación y desarroIlo $(I+D)$, es seguida por una fase de desarrollo y finalmente arriba a las etapas de producción y comercialización. Identifica la innovación con actividades formales de I+D. Las capacidades tecnológicas de una organización o sociedad son esencialmente función de las fronteras de sus conocimientos (López, 2004).

Como se puede observar en la figura 3 el conocimiento tecnológico se concibe como explícito, articulado, imitable, codificable y perfectamente transmisible. Las empresas pueden producir y usar innovaciones a partir de un banco de conocimientos que, según los casos, será o no de acceso gratuito, pero que siempre entregará una información codificada y fácilmente reproducible; se supone, por ende, que las tecnologías 
FIGURA 2

\section{Modelo de Henderson y Clark (1990)}

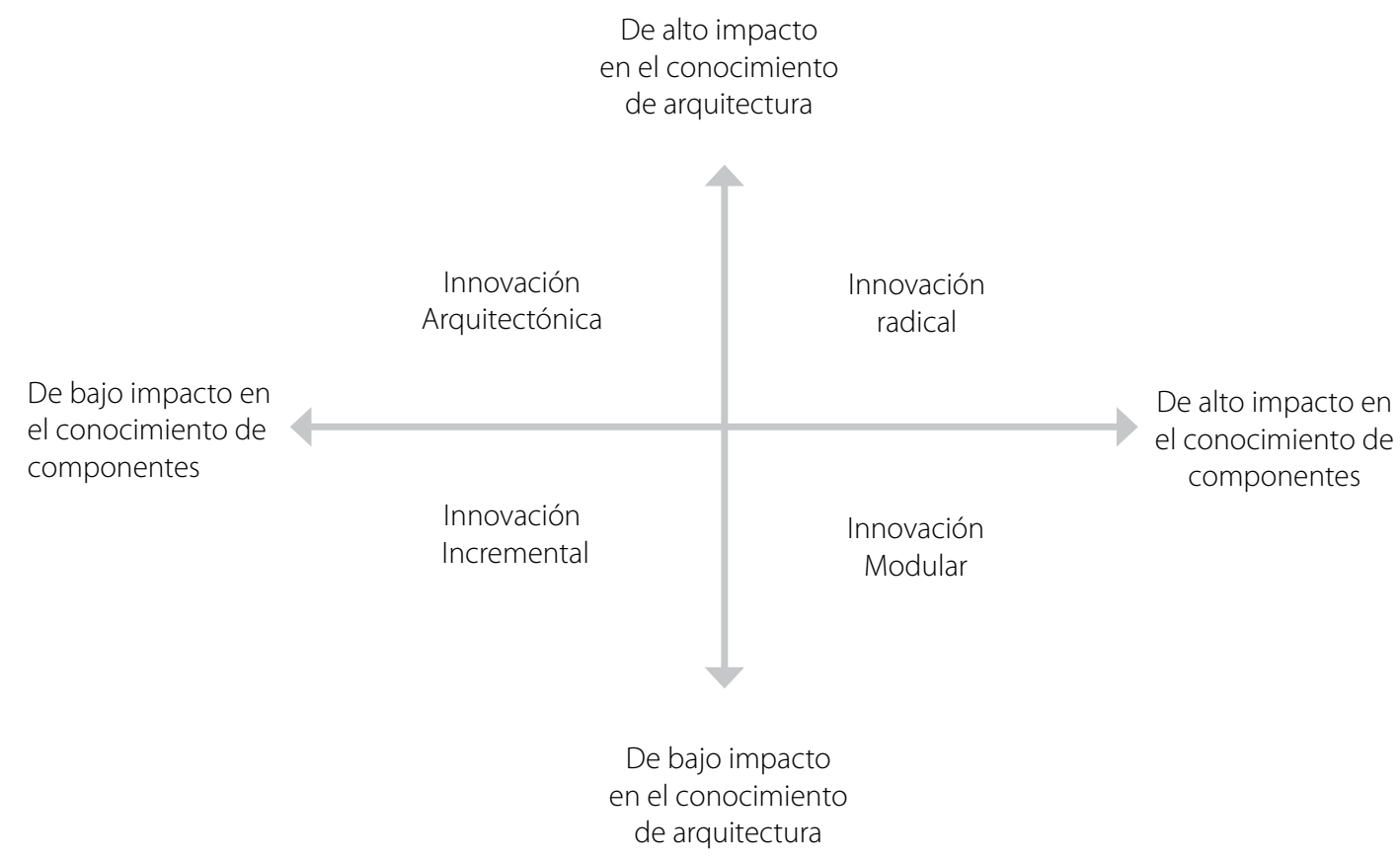

Fuente: Harris et al. (2000).

son usadas con el mismo grado de eficiencia en todas las empresas.

La tecnología se percibe como enteramente realizada con anterioridad a su incorporación a la esfera productiva (no hay retroalimentación proveniente de esta última) y, en general, se ignoran las innovaciones provenientes de actividades no formales. La ciencia, en tanto, se concibe como situada afuera del proceso económico.

En el Modelo interactivo del proceso de innovación -chain linked- (Ver figura 3) también conocida como modelo de innovación no lineal, se desvanece la distinción shumpeteriana entre invención, innovación y difusión como tres actos claramente separables a favor de una concepción del cambio tecnológico como proceso continuo. La información sube desde los escenarios "aguas abajo" (producción, comercialización y distribución) hacia los escenarios "aguas arriba" (invención y/o concepción analítica del producto o proceso). Se enfatizan las interacciones entre reflexión científica e innovación tecnológica en cada escenario del proceso de innovación, desde la invención hasta la comercialización.

Los procesos de aprendizaje tecnológico nunca son automáticos, pues son afectados por un buen número de variables entre las que se encuentra la orientación del aprendizaje, la base de conocimientos existente y la intensidad del esfuerzo por internalizar el conocimiento nuevo que implica la adquisición de una nueva tecnología. Las empresas aprenden de diversas maneras, y cada una de ellas lleva a mejorar en el stock 
Figura 3

\section{Dos modelos contrapuestos de la innovación}

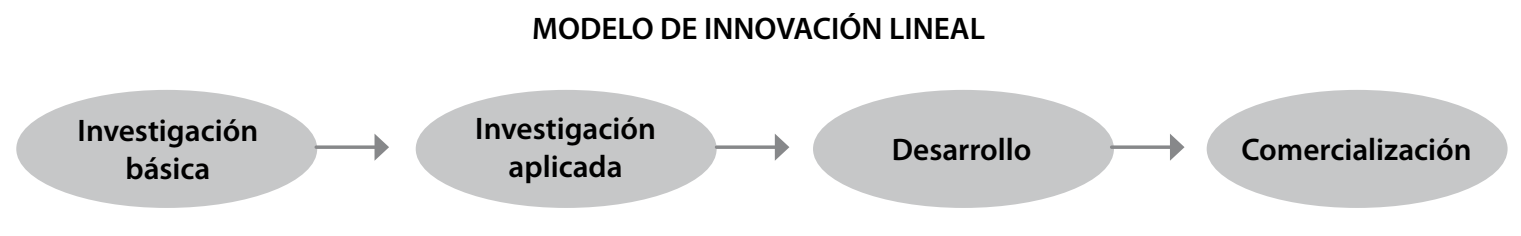

\section{MODELO DE INNOVACIÓN NO LINEAL}

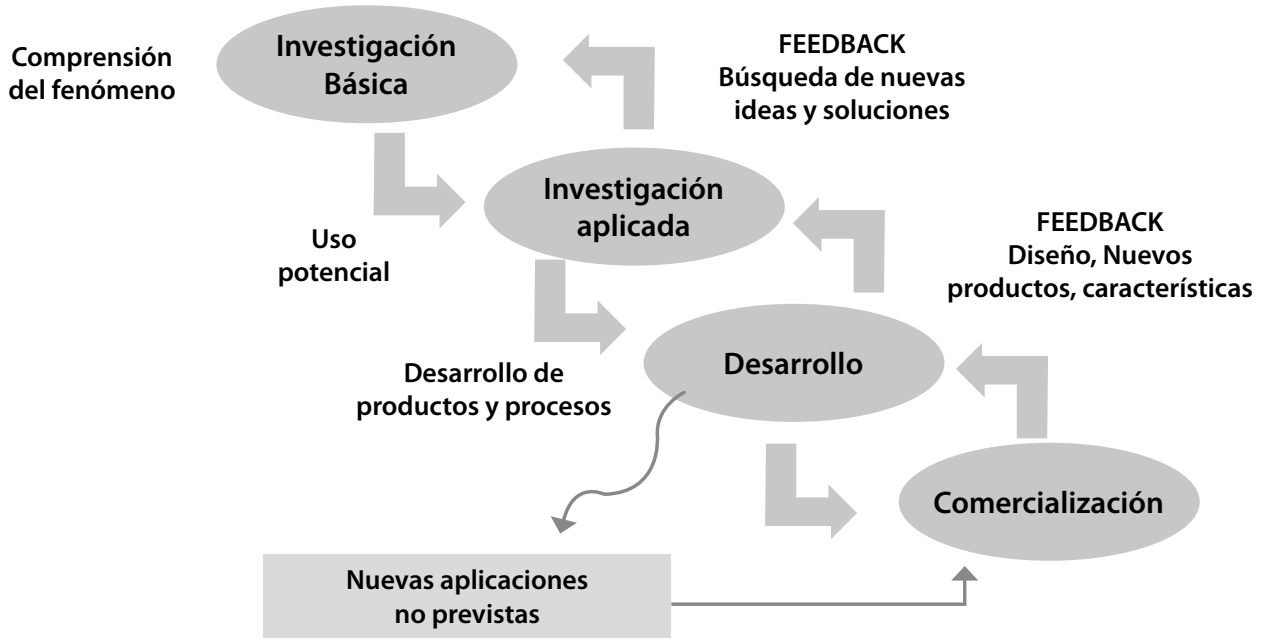

Fuente: Fuente: Cimoli, Perez \& Rovira (2010).

de conocimiento y sus capacidades tecnológicas específicas, lo que a su vez genera un rango de trayectorias de avance tecnológico y no una simple reducción promedio de costos. A su vez, los distintos tipos de aprendizaje abren diversas direcciones de cambio técnico incremental. En este modelo existe una constante retroalimentación entre cada uno de los componentes. Las innovaciones surgen de las demandas y necesidades de los consumidores.

El quinto Modelo corresponde a Teece (1983). Este autor propone un modelo que toma en cuenta dos factores que son importantes para lucrarse de una innovación: facilidad de ser imitada (imitabilidad) y activos complementarios.
La imitabilidad puede venir de la propiedad intelectual, protección de la tecnología o del hecho de que los imitadores no tienen las competencias para imitar la tecnología. Los activos complementarios son las capacidades -aparte de tecnologías- que la empresa necesita para explotar la innovación (manufactura, mercadeo, canales de distribución, servicio, reputación, marca y tecnologías complementarias).

El sexto y último modelo, presentado por Escorsa (2003), corresponde al de Kline quien en 1985, identifica cinco caminos o trayectorias que conducen a la innovación, todos importantes que se pueden apreciar en la figura No. 4. El camino central de la innovación empieza con una idea 
que se materializa en un invento y/o diseño analítico, el cual evidentemente, ha de responder a una necesidad del mercado. El diseño analítico se denomina también diseño de ingeniería. Este invento o diseño analítico pasa a continuación por un proceso de diseño detallado que acaba en un prototipo, que es probado en la fase de desarrollo tecnológico. Más adelante vienen las etapas de fabricación y comercialización.

Existen diversas retroalimentaciones a) entre cada etapa del camino central y la etapa anterior; b) desde el producto final, que puede obligar a hacer correcciones por alguna deficiencia; c) desde el producto final hasta el mercado potencial, cada nuevo producto crea nuevas condiciones del mercado.

El modelo de Kline relaciona la ciencia con la tecnología en todas las partes del modelo y no solo en las etapas iniciales. Considera la innovación como una manera de encontrar y solucionar problemas.

\section{Sistema nacional de innovación}

Un sistema nacional de innovación está constituido por redes locales, relacionadas o no entre ellas, en relación o no con las redes mundiales. De acuerdo con Nelson (1993) constituyen un conjunto de instituciones cuyas interacciones determinan el desempeño innovador de las empresas.

Según la definición que CEPAL (2002) le da a un Sistema de Innovación, “Es el conjunto de agentes, instituciones y normas en el que se apoyan los procesos de incorporación de tecnología, generalmente sistemas nacionales de innovación, que determinan el ritmo de generación, adaptación, adquisición y difusión de conocimientos tecnológicos en todas las actividades productivas" (p. 12).

Un Sistema Nacional de Innovación SNI, también es entendido como el conjunto de instituciones que conjunta y/o individualmente contribuyen

\section{Modelo de Innovación de Kline}

\section{MODELO DE KLINE}

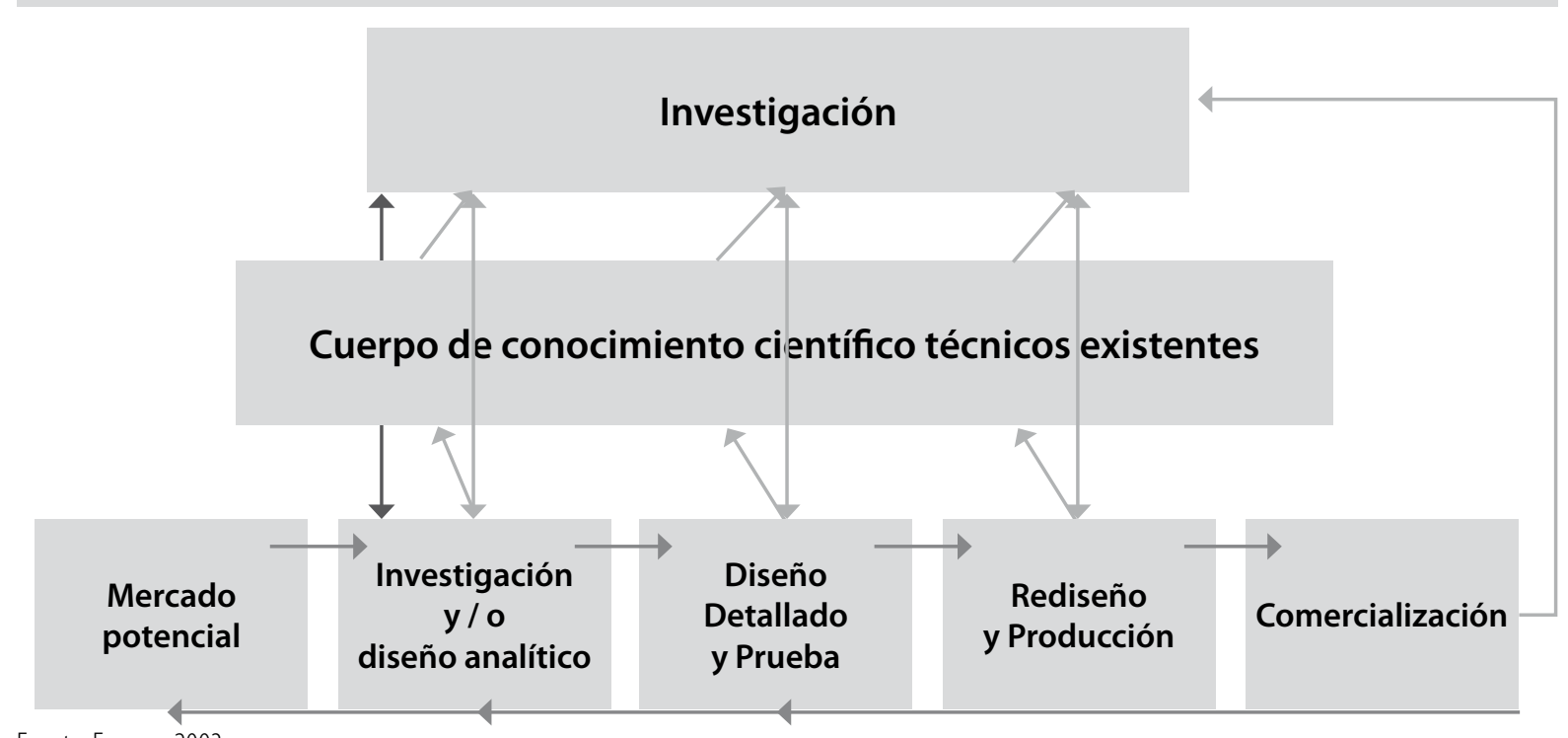

Fuente: Escorsa, 2003. 
al desarrollo y la difusión de nuevas tecnologías y las estructuras políticas que los gobiernos implementan para influir en el proceso de innovación. Como tal es un sistema de instituciones interconectadas para crear, almacenar y transferir el conocimiento, las habilidades y artefactos que definen nuevas tecnologías.

El concepto de Sistema Nacional de Innovación (SNI) fue introducido por Freeman y Pérez (1988), su principal foco de análisis es la interacción entre los agentes económico, social y político del sistema, que favorecen el desarrollo de capacidades y de la difusión de las innovaciones (Rovira, 2011).
Como puede observarse en la Figura 5, para que el sistema funcione adecuadamente, es necesario que exista una buena interrelación entre cada uno de sus componentes. Las empresas actúan como los actores de mercado que requieren de recursos financieros y de generación de conocimientos que pueden ser proveídos por las instituciones de Educación Superior y los centros de investigación especializados, mientras que el Estado participa dentro de este sistema a través de instituciones de gobierno propiciando una plataforma adecuada con reglas del juego claras y que den el marco legal adecuado.

\section{El SNI: Componentes y relaciones}

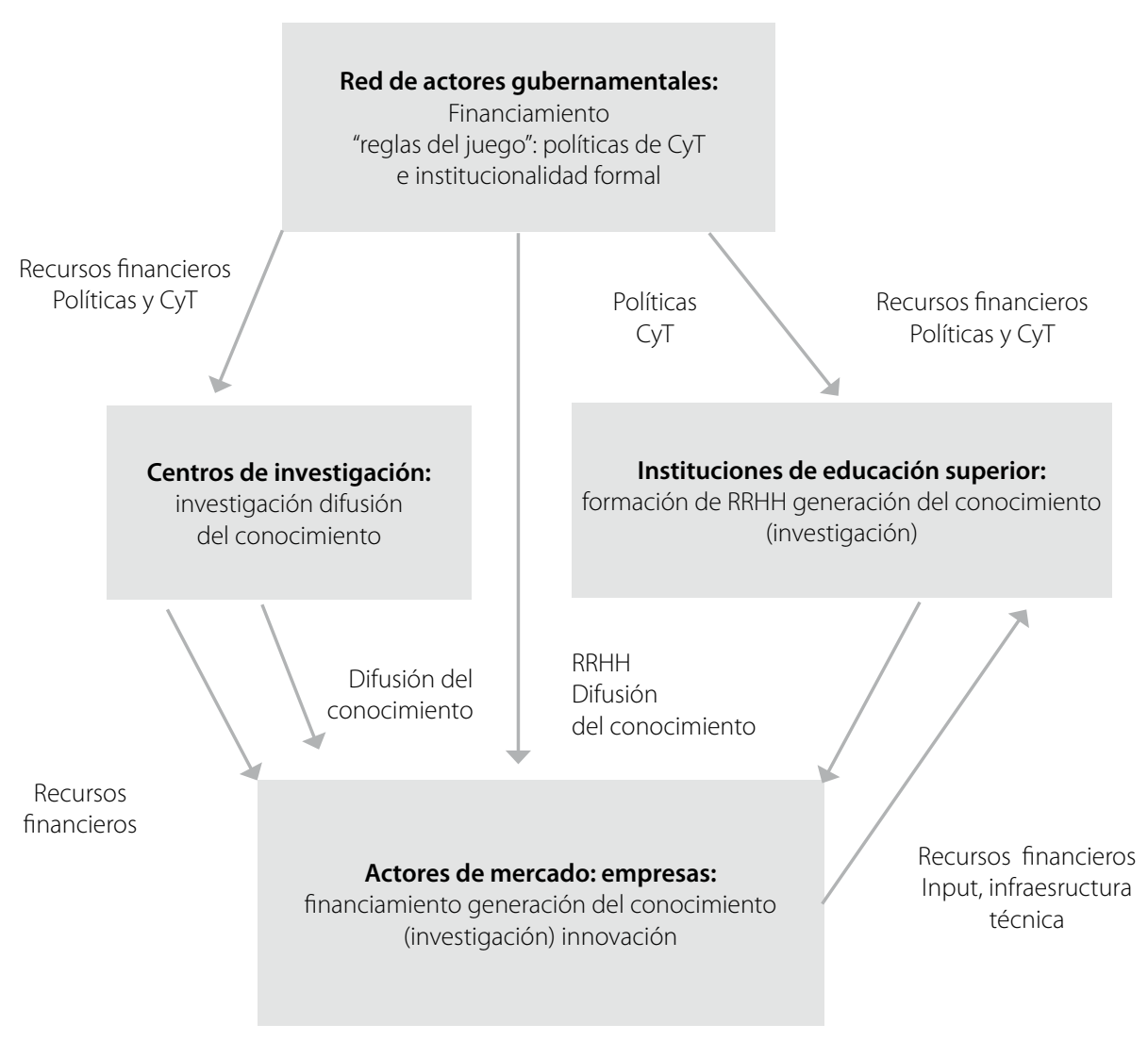

Fuente: Cimoli, Perez \& Rovira (2010). 
Pero ¿por qué hablar de Sistema Nacional de Innovación?, porque la mayor parte de micro, pequeñas y medianas empresas no cuenta con los recursos ni la infraestructura adecuada para generar conocimiento que le permita realizar cambios sustanciales e innovadores en sus productos y/o servicios, por lo tanto debe aliarse con las Instituciones de Educación Superior y Centros de Investigación Especializados mediante convenios y acuerdos donde los estudiantes puedan desarrollar investigaciones aplicadas útiles para lograr innovaciones trascendentales. Por su parte, los actores gubernamentales deben apoyar con financiamiento y el establecimiento de políticas de innovación y emprendimiento claras. Como puede observarse en la Figura 6, el financiamiento para las empresas le corresponde tanto al sector gubernamental como a las mismas empresas, la formación de recursos humanos le corresponde a las Instituciones de Educación Superior, la generación del conocimiento a través de investigación básica y aplicada es función de los centros de investigación, las Instituciones de Educación Superior y las empresas. Por último la innovación le corresponde como función a las empresas.
El SNI es una red de agentes y sus interacciones que están directa o indirectamente relacionados con la introducción y/o difusión de nuevos productos y nuevos procesos tecnológicos en una economía. Esta red está constituida no solo por los agentes públicos, los que pueden desarrollar o financiar la innovación tecnológica, sino que también por todos aquellos que participan en las diferentes etapas de dicho proceso innovativo en el ámbito privado.

\section{Gestión de la innovación}

La gestión de la innovación tiene como objetivo organizar los recursos disponibles en la empresa (humanos, técnicos y económicos), con el fin de generar conocimiento traducido en nuevos productos, mejora en los productos existentes, tomando en cuenta las etapas de fabricación y comercialización.

Para garantizar que la gestión de la innovación sea exitosa es importante cumplir con las siguientes funciones básicas según Dornberger, Suvelza \& Bernal (2011):

FIGURA 6

Principales funciones de los agentes del SNI en la generación, difusión y uso del conocimiento

\begin{tabular}{|c|c|c|c|c|c|}
\hline \multirow[b]{2}{*}{ Funciones } & & \multicolumn{4}{|c|}{ Actores del SNI } \\
\hline & & $\begin{array}{c}\text { Actores } \\
\text { gubernamentales }\end{array}$ & $\begin{array}{c}\text { Centros de } \\
\text { investigación }\end{array}$ & $\begin{array}{l}\text { Instituciones } \\
\text { de educación } \\
\text { superior }\end{array}$ & $\begin{array}{l}\text { Actores de } \\
\text { mercado: } \\
\text { empresas }\end{array}$ \\
\hline \multicolumn{2}{|l|}{ Financiamiento } & $\mathbf{x}$ & & & $\mathbf{x}$ \\
\hline \multicolumn{2}{|c|}{ Formación RRHH pata las capacidades CyT } & & & $\mathbf{x}$ & \\
\hline \multirow{2}{*}{ Generación del conocimiento CyT } & Investigación Básica & & & $\mathbf{x}$ & \\
\hline & Investigación Aplicada & & $\mathbf{x}$ & $\mathbf{x}$ & $\mathbf{x}$ \\
\hline \multicolumn{2}{|l|}{ Difusión del conocimiento CyT } & & $\mathbf{x}$ & $\mathbf{x}$ & \\
\hline \multicolumn{2}{|l|}{ Innovación } & & & & $\mathbf{x}$ \\
\hline
\end{tabular}

Fuente: Cimoli, Perez \& Rovira (2010). 
a) Inventariar: se debe conocer las capacidades tecnológicas con las que cuenta la empresa.

b) Vigilar: estar alerta sobre la evolución de la nueva tecnología. Vigilancia de la tecnología de los competidores.

c) Evaluar: se debe determinar la competitividad y el potencial tecnológico propio, estudiar posibles estrategias.

d) Enriquecer: aumentar el patrimonio de la empresa a través de la inversión en tecnología propia, ajena o mixta.

e) Optimizar: emplear los recursos de la mejor manera posible.

f) Protección de las innovaciones propias y actualización constante de los conocimientos.

Se debe tener claridad que las actividades de innovación conllevan todas las decisiones y desarrollos científicos, tecnológicos, organizacionales, financieros y comerciales que se llevan a cabo dentro de las empresas, incluyendo las inversiones en nuevos conocimientos.

De acuerdo con Ponti (2010), se puede construir o reinventar una empresa innovadora si se aplican estos siete movimientos que contribuirán a gestionar de forma significativa la innovación dentro de las empresas.

a) Saber dónde se quiere ir, es decir, cuál es el rumbo que se le quiere dar a la empresa, involucra tener ideas claras, objetivos claros, misión, visión y valores de la empresa relacionados con el proceso de innovación, construir un modelo de negocio que defina con claridad el segmento de mercado y los clientes, que producto o servicio se ofrece, cuál es el valor agregado que tiene, de qué forma se venderá y qué canales de distribución se utilizarán. Pero sobre todo cómo la empresa se diferenciará de los competidores, cuál es la diferencia que más valoran los clientes. b) El segundo movimiento está dirigido a la conformación de un equipo de trabajo, en involucrar a todo el personal en el Comité de Innovación $(\mathrm{Cl})$, que será el órgano de gestión de la innovación. El Cl debe estar compuesto por personas creativas, motivadas hacia la innovación, con resultados claros, comprometidas, apasionadas.

c) El cambio, es el tercer movimiento, revolucionar las estructuras internas y el contacto con el exterior. Si una empresa está demasiado jerarquizada tendrá importantes dificultades para innovar. Así mismo se debe propiciar la comunicación entre los diferentes departamentos de la empresa, se debe crear una cultura de la conversación.

d) El cuarto movimiento invita a evaluar las tendencias, a anticiparnos al futuro, a tener una visión prospectiva creando plataformas de reflexión y generando espacios para el pensamiento.

e) Desarrollar la creatividad fomentando el ingenio colectivo para lograr equipos de trabajo que tengan muy claro lo que debe hacerse, se identifiquen con la misión, logren un alto nivel de cohesión o integración, se ayuden entre sí y sientan empatía unos con otros.

f) Se deben generar bancos de proyectos innovadores con el objeto de lograr el financiamiento.

g) Por último, pero no menos importante, se debe sistematizar la innovación, es decir, medir los resultados, socializarlos y promover la cultura de la innovación.

Jaramillo et al. (2001) indican que la adquisición y generación de conocimiento nuevo o de relevancia para la empresa se debe gestionar de forma adecuada mediante: 
a) El desarrollo de investigación y experimentación que comprende el trabajo creativo que se emprende con el fin de incrementar el stock de conocimientos. Aquí se incluye la construcción y prueba de prototipos de un nuevo producto o proceso.

b) Adquisición de tecnología incorporada, que incluye maquinaria y equipo que favorecerá el desempeño de la empresa.

c) Modernización de los equipos e ingeniería industrial, que involucra cambios en la producción y en los procesos de control de calidad, métodos, patrones y la inclusión de software.

d) Adquisición de edificios, herramientas y equipos que no tienen un mejor desempeño tecnológico pero que son necesarios para la implementación de productos nuevos o mejorados.

e) Readaptación de personal en las nuevas técnicas o en el uso de nueva maquinaria.

f) Mercadeo de productos nuevos o mejorados, que incluye investigación preliminar de mercado y publicidad para el lanzamiento.

La innovación tecnológica se produce mediante los esfuerzos técnicos desarrollados dentro de la empresa, pero con una gran interacción con el entorno exterior. Es de vital importancia la búsqueda proactiva de información obtenida de fuentes externas, donde se determinen las necesidades de los clientes y la actividad de la competencia.

\section{Innovación y estrategia}

El concepto de estrategia se usa normalmente para designar los medios empleados en la obtención de cierto fin, involucra la racionalidad orientada a un objetivo; es utilizado para designar la manera en la cual una persona actúa en cierta circunstancia de acuerdo con lo que piensa, buscando tener ventajas sobre los otros; finalmente es utilizado para designar procedimientos usados en una situación de confrontación con el fin de privar al oponente de sus medios de lucha y obligarlo a abandonar el combate, es decir los medios destinados para obtener la victoria (Deleuze,1987).

La estrategia de innovación según Hamel (1998) es la capacidad de reciclarse que existe en los distintos modelos de industria y a partir de los cuales se crea valor para los clientes. Se proporciona a los clientes nuevos productos lo cual genera utilidades para los accionistas. Para Markides (1999) la estrategia de innovación es una re-conceptualización fundamental que se da en las empresas a través de la cual las organizaciones son capaces de reinventar la forma de jugar en el tablero empresarial.

Schlegelmilch (2003) indica que es la re-conceptualización del modelo de empresa (rompiendo los roles establecidos y cambiando la forma de competir) logrando un mayor valor para los clientes y crecimiento para la empresa. Según Champman (2007) a través de la estrategia de innovación se van marcando metas a conseguir por las empresas.

La consistencia estratégica se da cuando las acciones de una empresa son coherentes con las expectativas de la dirección y estas a su vez lo son con el mercado y su entorno.

\section{Estrategia competitiva}

Porter (1980) describió la estrategia competitiva como las acciones ofensivas o defensivas de una empresa para crear una posición defendible dentro de una industria, acciones que son la respuesta a las cinco fuerzas competitivas que indicó como determinantes de la naturaleza y el grado de competencia que rodea a una empresa y que como resultado, busca obtener un importante rendimiento sobre la inversión. Por- 
ter identificó tres categorías genéricas que podían usarse individualmente o en conjunto, para crear en el largo plazo esa posición defendible que sobrepasara el desempeño de los competidores en una industria. Esas tres estrategias genéricas son:

a) El liderazgo en costos totales bajos

Estrategia muy popular en la década de los 70's, debido al concepto muy arraigado de la curva de experiencia. Mantener el costo más bajo frente a los competidores y lograr un volumen alto de ventas era el tema central de la estrategia. Por lo tanto la calidad, el servicio, la reducción de costos mediante una mayor experiencia, la construcción eficiente de economías de escala, el rígido control de costos particularmente de los costos variables. Se buscaba la minimización de costos en las áreas de investigación y desarrollo, fuerza de ventas, publicidad, personal y en general en cada área de la operación de la empresa.

Si la empresa tenía una posición de costos bajos, se esperaba que esto la condujera a obtener utilidades por encima del promedio de la industria y la protegiera de las cinco fuerzas competitivas. En la medida en que los competidores luchaban mediante rebajas de precio, sus utilidades se erosionaban hasta que aquellos que quedaban en el nivel más próximo al competidor más eficiente eran eliminados. Los competidores menos eficientes eran los primeros en sufrir las presiones competitivas. Lograr una posición de costo total bajo, frecuentemente requería una alta participación relativa de mercado (se refiere a la participación en el mercado de una empresa con relación a su competidor más importante) u otro tipo de ventaja, como podría ser el acceso a las materias primas.
Podría exigir también un diseño del producto que facilitara su fabricación, mantener una amplia línea de productos relacionados para distribuir entre ellos el costo, así como servir a los segmentos más grandes de clientes para asegurar volumen de ventas. Como contraposición, implementar una estrategia de costo bajo podría implicar grandes inversiones de capital en tecnología de punta, precios agresivos y reducir los márgenes de utilidad para comprar una mayor participación en el mercado. Por aquella época, la estrategia de liderazgo en costo bajo fue el fundamento del éxito de compañías como Briggs \& Stratton Corp., Texas Instruments, Black \& Decker y Du Pont.

b) La diferenciación

Una segunda estrategia es la de crearle al producto o servicio algo que sea percibido en toda la industria como único. La diferenciación se considera como la barrera protectora contra la competencia debido a la lealtad de marca, la que, como resultante, debería producir una menor sensibilidad al precio. Diferenciarse significa sacrificar participación de mercado e involucrarse en actividades costosas como investigación, diseño del producto, materiales de alta calidad o incrementar el servicio al cliente. Sin embargo, esta situación de incompatibilidad con la estrategia de liderazgo de costos bajos no se daba en todas las industrias y existían negocios que podían competir con costos bajos y precios comparables a los de la competencia. Compañías que se distinguieron en su momento por adoptar alguna forma de diferenciación fueron: Mercedes-Benz (diseño e imagen de marca), Caterpillar (red de distribución) y Coleman (tecnología), entre muchas otras. 
c) El enfoque

La tercera estrategia consiste en concentrarse en un grupo específico de clientes, en un segmento de la línea de productos o en un mercado geográfico. La estrategia se basa en la premisa de que la empresa está en condiciones de servir a un objetivo estratégico más reducido en forma más eficiente que los competidores de amplia cobertura. Como resultado, la empresa se diferenciaba al atender mejor las necesidades de un mercado-meta específico, o reduciendo costos sirviendo a ese mercado, o ambas cosas. The Martin-Brower Co. uno de los grandes distribuidores de alimentos en los Estados Unidos, fue un ejemplo en la adopción de la estrategia de enfoque limitando su servicio solamente a las ocho principales cadenas de restaurantes de comida rápida. Las tres estrategias genéricas de Porter eran alternativas, maneras viables de enfrentar a las fuerzas competitivas. La empresa que fallara en desarrollar su estrategia en por lo menos una de estas directrices, quedaba atrapada en el centro, como la empresa $\mathrm{C}$ de la figura, situada en una posición estratégica extremadamente pobre (una empresa con precio alto para productos percibidos como de baja calidad). Porter describe a este tipo de empresa con fallas en su participación de mercado, en su inversión de capital y con limitada capacidad de maniobra para ejecutar la estrategia del bajo costo, de la diferenciación o del enfoque.

\section{Estrategias competitivas basadas en la innovación}

De acuerdo con Caballano (2010) las estrategias competitivas basadas en la innovación se clasifican en:

a) Estrategia innovadora ofensiva: Aquí se ubican las empresas que pretenden conseguir la supremacía tecnológica en el mercado mediante la comercialización permanente de nuevos productos, asumiendo un gran riesgo, esperando una gran rentabilidad, existe mucha iniciativa empresarial.

b) Estrategia innovadora defensiva: Siguen el camino del líder en tecnología, suelen ser los líderes económicos del mercado, no son propensas a realizar innovaciones radicales.

c) Estrategia imitativa: El grado de innovación es mínimo, se contentan con seguir a los líderes tecnológicos, deben tener el mercado cautivo, menores costos y elevada eficiencia directiva.

d) Estrategia oportunista: Buscan el punto más débil del competidor y allí enfocan sus esfuerzos.

e) Estrategia dependiente: Es dependiente cuando las ventas se concentran en pocos clientes o el capital es controlado por otra empresa. La utilizan las pequeñas empresas cuando logran ser subcontratadas por las grandes empresas.

f) Estrategia tradicional: Las utilizan las empresas cuando sus productos permanecen inamovibles desde el punto de vista funcional. Se basa en saberes artesanales y su input científico es mínimo o nulo. La estrategia tradicional no es innovadora.

\section{CONCLUSIONES}

La innovación es un proceso empresarial que se relaciona con cambio de procesos productivos, renovación tecnológica, desarrollo organizacional, comercialización, estrategia, método, que si se gestiona adecuadamente contribuirá en forma considerable a lograr el crecimiento y desarrollo de las empresas, sectores, países y regiones. 
El proceso de generación del conocimiento no es lineal, se basa en ensayos de prueba y error, presenta discontinuidades y especificidades, por tal motivo seguir un modelo lineal de innovación no responde a las demandas del mercado. Por lo tanto es fundamental una mayor integración de los intereses políticos, académicos e industriales en la conducción, gestión y regulación de la innovación.

Es indispensable conformar los Sistemas Nacionales de Innovación con una buena interrelación entre cada uno de sus componentes, las empresas actuando como los actores de mercado que requieren de recursos financieros y generación de conocimientos que pueden ser proveídos por las instituciones de Educación Superior y los Centros de investigación Especializados; mientras que el Estado participa dentro de este sistema a través de instituciones de gobierno propiciando una plataforma adecuada con reglas del juego claras y estableciendo el marco legal pertinente.

Y finalmente, la micro, pequeña y mediana empresa está obligada a innovar o de lo contrario desaparecerá, deberá aprovechar ideas nuevas estructurándolas adecuadamente, orientando los esfuerzos a satisfacer las demandas de un mercado cada vez más exigente y que busca productos y servicios novedosos, deberá estar dispuesta a buscar oportunidades de mercado, replanteando constantemente los objetivos de la empresa, tomando en cuenta que lo que hoy puede ser una fortaleza, puede convertirse pronto en una debilidad si no se está dispuesto al cambio.

\section{REFERENCIAS}

Afuah, A. (2009). Innovation models. En V.K. Narayanan \& Gina O'Connor, Encyclopedia of Technology and Innovation Management.

Afuah, A. (2009). Strategic Innovation: New game strategies for competitive Advantage. Routledg.
Albernathy, W. \& Clark, K. (1985). Mapeo de los vientos de la destrucción creativa.Technology review,

Chapman R. (2007) How strategic innovation really gets started. VOL. 35 NO. 1 pp. 21-29, Q Emerald Group Publishing Limited, STRATEGY \& LEADERSHIP Recuperado el 12 de marzo 2011 en:http://www.rp.edu.sg/cie/ enewsletter_Jan2007/index/article.pdf

Cimoli, M. Perez, W \& Rovira, S. (2010) Innovar para crecer: Desafíos y oportunidades para el desarrollo sostenible e inclusivo en Iberoamérica. Chile: CEPAL/SEGIB

Damampour, F. \& Evan, W. (1996). Organizational innovation and performance; The problem of organizational lag. Administrative Sience Quarterly, 29, 392-409.

Damampour, F. (1987). The adoption of technological, administrative and ancillary innovations. Journal of management, 13, 675-688.

Damampour, F. \& Evan, W. (1999). The relationship between types of innovation and organizational performance.

Deleuze, G. (1987). Foucault. Barcelona, España: Paidos Ibérica.

Díaz, M. (1996). Factores determinantes de la innovación tecnológica para las pequeñas empresas. Recuperado el 19 de julio, 2010 de:http://revistas.ucm.es/ emp/11316985/articulos/CESE9696110145A.PDF

Domenech, E. (2009). El futuro no lo escribirá un ingeniero, sino la respuesta de una necesidad latente. Revista Barcelona Innova. Recuperado el 12 de julio, 2010 de: http://w3.bcn.es/ AB_CDA/P00Persistencia/P00ServeiPersistenciaC tl/0,3259,83057194_83070530_2_234027407,00.html ?accio=detall\&nomtipusMCM=entrevista\&calMarc=1

Dornberger, U., Suvelza, A. \& Bernal, L. (2011). Gestión de la fase temprana de la innovación. Alemania: SEPT, Working paper series.

Drucker, P. (1954). The Practice of managment. EE.UU: Butterworth-Heinemann.

Drucker, P. (2002). Innovación y emprendimiento. New York, EE.UU.: Harper Business.

Escorsa, P. (2003). Tecnología e innovación en la Empresa. Barcelona, España. Recuperado el 12 de febrero, 2011 de: http://books.google.com.gt/books? 
Freeman, C. \& Pérez, C. (1988). Structural crises of adjustment, business cycles and investment behavior", Thechnical Change and Economic Theory, Londres: Pinter Publisher.

Hamel, G. \& Phahalad, C.K. (1996). Competing for the future. EE.UU, Harvard: Business School Press.

Harris, Coles \& Dickson (2000). Innovación arquitectónica: la reconfiguración de las tecnologías de productos existentes y el fracaso de las empresas ya establecidas.

Henderson, R. \& Clark, K. (1990). La innovación arquitectónica: la reconfiguración de las tecnologías de productos existentes y el fracaso de las empresas establecidas. Ciencias Administrativas. Recuperado el 12 de julio, 2010 de: http://innovationzen.com/blog/2006/08/11/ innovation-management-theory-part-3/

Hidalgo, A. \& Vizán, A. (2008). Los Factores claves de la innovación tecnológica: Claves de Competitividad Empresarial. Revista Dirección, organización y administración de empresas. España: Editorial Universidad Politécnica de Madrid.

Izque, (2005). Transilience maps. Recuperado de http:// www.provenmodels.com/571

Jaramillo, H. Lugones, G. \& Salazar, M. (2001). Manual de Bogotá: Normalización de Indicadores de Innovación Tecnológica en América Latina y el Caribe RICYT, OEA, CYTED. Colombia.

Jiménez, D. \& Sanz, R. (2004). Innovation and human resource management fit: An empirical study. International Journal of Manpower, 26(4), 364-381.

Kline, (1985). Tecnología e innovación en la Empresa. Barcelona, España:

López, G. (2004). La innovación: un proceso socialmente distribuido. Scientia et Technica, 25, 197-202.

Llobet, F. (2006). Innovación y economías estratégicas. Recuperado el 12 de mayo, 2010 de: http://www.innovationthink.com/innovacion/2006/03/innovacion_y_ economias_estrate.html\#more

Márquez, A. \& Pérez, L. (2007). Estudio de los factores intervinientes en el proceso innovativo de las Pyme metalmecánicas. Espacios, 28(2), 5-20.
Markides, C. (1999). En la Estrategia está el éxito. Colombia: Editorial Normal.

Martín-Hidalgo, L. (2009). Sistema de gestión de la innovación en las empresas. Guatemala: Serviprensa.

Nelson, R. (1993). National Innovation System: a Comparative Analysis, Nueva York: Oxford University Press.

Ponti, F. (2010). Los siete movimientos de la innovación. Colombia: Grupo Editorial Norma.

Porter, M. (1980). Competitive Strategy. New York, Estados Unidos: Free Press. Recuperado el 15 de marzo, 2011 de: http://drfd.hbs.edu/fit/public/facultylnfo. do?faclnfo=ovr\&facld $=6532$

Porter, M. (1999). Estrategia Competitiva. Técnicas para el análisis de los sectores industriales y la competencia. México: Compañía Editorial Continental.

Porter, M. (2005). Cómo las Fuerzas Competitivas le dan forma a la Estrategia.

Pujol, R. (2005). Guía práctica de la innovación para PYMES. España: Anetcom.

Rovira, S. (2011). Taller Innovación para el desarrollo: Experiencias, políticas y retos para América Latina y el Caribe. República Dominicana: CEPAL.

Schlegelmilch, B. \& Keegan W. (2003). Global Marketing Management: A European Perspective. Prentice-Hall.

Schumpeter, J. (1912). The Theory of Economic Development: An inquiry into profits, capital, credit, interest and the business cycle. Estados Unidos: Transaction Publishers.

Schumpeter. J. (1939). Business Cycles: A theoretical, historical and statistical analysis of the Capitalist process. Nueva York, Toronto, Londres: McGraw-Hill.

Tanaka, N., Glaude, M. \& Gault, F. (2005). Manual de Oslo. Normalización de Indicadores de innovación tecnológica OCDE. Francia.

West, M. \& Farr, J. (1990). Innovation and creativity at work. Londres: Editorial John Wiley.

Recibido: 15-06-2011

Aceptado: 02-11-2011 